\title{
Quantum-assisted photoelectric gain effects in perovskite solar cells
}

\author{
Ying-Chiao Wang $\mathbb{D}^{1}$, Shao-Ku Huang ${ }^{2}$, Toshihiro Nakamura ${ }^{3}$, Yu-Ting Kao ${ }^{4}$, Chun-Hao Chiang ${ }^{2}$, Di-Yan Wang ${ }^{4}$, \\ Yuan Jay Chang ${ }^{4}$, Nobuyoshi Koshida ${ }^{5}$, Toshikazu Shimada ${ }^{5}$, Shihao Liu', Chun-Wei Chen $\mathbb{0}^{2,6}$ and \\ Kazuhito Tsukagoshi (id)
}

\begin{abstract}
Further boosting the power conversion efficiencies (PCEs) of perovskite solar cells (PSCs) without excessively increasing production expenses is critical for practical applications. Here, we introduce silicon quantum dots (SiQDs) to enable perovskites to harvest additional sunlight without changing PSC processes. These SiQDs can convert shorter wavelength excitation light $(300-530 \mathrm{~nm})$ into visible region light and reflect longer wavelength perovskiteunabsorbed visible light (550-800 nm), leading to broadband light absorption enhancement in PSCs. As a result, the SiQD-based photocurrent gain can improve the external quantum efficiencies of PSCs over a wide wavelength range of 360-760 nm, yielding relatively enhanced short-circuit current density $\left(+1.66 \mathrm{~mA} / \mathrm{cm}^{2}\right)$ and PCE $(+1.4 \%)$. Surprisingly, even the PSC with a low-purity perovskite layer shows an ultrahigh PCE improvement of 5.6\%. Our findings demonstrate QD-assisted effects based on earth-abundant and environmentally friendly silicon, leading to effective optical management that remarkably promotes the performance of PSCs and enables the balance of costs to be substantially addressed.
\end{abstract}

\section{Introduction}

The past decade has witnessed amazing advances in organic-inorganic perovskite solar cells (PSCs), with the power conversion efficiency (PCE) drastically increasing from $3.8 \%$ to more than $25 \%{ }^{1-10}$. The extraordinary PCEs have already exceeded those of multicrystalline $\mathrm{Si}$ solar cells and are not far from the single crystal values ${ }^{10}$. Even so, crystalline $\mathrm{Si}$ modules still dominate the current photovoltaic industry because their mature technology has led to a continuous decrease in $\operatorname{costs}^{11}$. To date, the major costs for solar panel manufacturing are area-related balance-of-system costs; thus, increasing the PCE is a key

\footnotetext{
Correspondence: Chun-Wei Chen (chunwei@ntu.edu.tw) or

Kazuhito Tsukagoshi (TSUKAGOSHI.Kazuhito@nims.go.jp)

${ }^{1}$ International Center for Young Scientists (ICYS) \& WPI International Center for Materials Nanoarchitectonics (WPI-MANA), National Institute for Materials

Science (NIMS), Tsukuba, Ibaraki 305-0044, Japan

${ }^{2}$ Department of Materials Science and Engineering, National Taiwan University, Taipei 10617, Taiwan

Full list of author information is available at the end of the article.

These authors contributed equally: Ying-Chiao Wang, Shao-Ku Huang
}

direction for reducing the levelized costs of solar energy $^{11-15}$. Unfortunately, the PCEs of single-junction solar cells are limited to the Shockley-Queisser limit due to the thermal relaxation of photocarriers with energies in excess of the bandgap energies (known as thermalization loss) $)^{11-17}$. Multijunction solar cells offer an effective solution to broaden the sunlight-sensitive region by utilizing multiple light harvesters with various absorption bands. A recent study on the perovskite (wide bandgap)/Si (narrow bandgap) tandem solar cell realized a certified PCE of $28.0 \%^{10}$, which once again places perovskite photovoltaics on the list of commercial candidates. However, to fabricate efficient perovskite/Si tandem photovoltaics, the upper perovskite crystals need to be well tailored to produce ultratransparent properties at long wavelengths while achieving excellent stability ${ }^{18}$. Therefore, the development of a simple method through which sunlight can be maximally absorbed to boost the performance of PSCs without changing the crystallization 
of the perovskite and without excessive material costs remains a challenge.

Silicon quantum dots (SiQDs) are fascinating materials for photovoltaics ${ }^{19-23}$ because of their superior properties, for example, strongly quantum-confined photoluminescence (PL) $)^{24,25}$ and color-tunable emission ${ }^{26,27}$, as well as their nontoxic and earth-abundant nature. Recently, we successfully synthesized colloidal SiQDs through pulsed ultraviolet (UV) laser illumination of porous Si powders ${ }^{28,29}$. With the introduction of hydrogen fluoride (HF), the dissolution of the surface oxide is more efficient, promoting surface passivation provided by hydrogen-terminated Si surfaces. Therefore, after hydrosilylation reactions, the PL quantum efficiencies of colloidal SiQDs with visible luminescence are increased.

To date, state-of-the-art PSCs require well-tailored high-quality crystals or custom-designed device structures to achieve high performance. In this work, multifunctional SiQDs synergistically boost the efficiencies of PSCs by fully capturing sunlight without changing the original PSC process. This quantum dot-assisted photovoltaic strategy uses facilely synthesized SiQDs to harvest the unabsorbed sunlight in PSCs, which has the costeffective production advantages of an earth-abundant element, a solution process and unchanged perovskite crystallization. The broadband spectral response of PSCs can be enhanced by harvesting the visible luminescence generated by SiQDs excited by short wavelength light between 300 and $530 \mathrm{~nm}$ and reflecting the perovskiteunabsorbed visible light in the $500-800 \mathrm{~nm}$ region, resulting in minimization of the thermalization loss. As a result, the SiQD-based PSC yields a promising PCE of $19.6 \%$, compared with $18.2 \%$ for the bare PSC device. The best-performing PSC reaches a short-circuit current density $\left(J_{\mathrm{SC}}\right)$ of $>23 \mathrm{~mA} / \mathrm{cm}^{2}$. Most impressively, using the SiQD-assisted photon management strategy, a low-quality PSC can exhibit an increase in the PCE from 7.3 to $12.9 \%$. This approach provides a simple pathway to improve the photocurrent generation in PSCs, giving an innovation method for realizing PSCs on the path toward commercialization.

\section{Materials and methods}

\section{SiQD synthesis procedures}

As shown in Supplementary Fig. 1, colloidal SiQDs were prepared by pulsed UV laser irradiation of porous $\mathrm{Si}$ (obtained from Quantum14, Ltd.) in a cosolvent (including 1-decene and $\mathrm{HF}$ ). Porous $\mathrm{Si}$ powder was prepared by standard electrochemical etching in a $\mathrm{HF} / \mathrm{C}_{2} \mathrm{H}_{5} \mathrm{OH}$ aqueous solution followed by drying under ambient conditions. To prepare the colloidal SiQDs, the porous $\mathrm{Si}$ powder was first dispersed in an organic solvent (such as 1-decence) in a quartz cuvette. Then, a small amount of aqueous HF solution was added to the organic solution.
Finally, $266 \mathrm{~nm}$ UV laser pulses from a Q-switched Nd: YAG laser (continuum) irradiated the sample with a pulse duration of $5 \mathrm{~ns}$ and a repetition rate of $15 \mathrm{~Hz}$ for $2 \mathrm{~h}$. The laser fluence was $\sim 0.2 \mathrm{~J} / \mathrm{cm}^{2}$. During laser irradiation, the porous Si powder dispersed in the HF/1-decene solution was constantly stirred by a magnetic stirrer. After laser irradiation, the supernatant of the liquid was filtered by centrifugation at $13,000 \mathrm{rpm}$ for $20 \mathrm{~min}$ using a membrane filter with a pore size of $\sim 200 \mathrm{~nm}$ to remove the HF and residual powder. This process enabled a colloidal SiQD solution in toluene to be obtained.

\section{Solar cell fabrication}

A $\mathrm{SnO}_{2}$ solution (Alfa Aesar, tin(IV) oxide, $15 \%$ in a $\mathrm{H}_{2} \mathrm{O}$ colloidal dispersion) was diluted with water to $2.67 \%$. The solution was then spin-coated on clean ITO glass at $3000 \mathrm{rpm}$ for $30 \mathrm{~s}$. The coated ITO substrate was annealed at $150^{\circ} \mathrm{C}$ for $30 \mathrm{~min}$ in air and then transferred to a glovebox. Solutions of $\mathrm{C}_{60}$ pyrrolidine tris-acid (CPTA) (Sigma-Aldrich, 97\%) in dimethylformamide (DMF) at a concentration of $2 \mathrm{mg} / \mathrm{ml}$ were prepared. The $\mathrm{ITO} / \mathrm{SnO}_{2}$ substrates were spin-coated with the CPTA solutions at $4000 \mathrm{rpm}$ for $30 \mathrm{~s}$ and then annealed at $140{ }^{\circ} \mathrm{C}$ for $10 \mathrm{~min}$ to facilitate esterification between the carboxyl groups of CPTA and the hydroxyl groups of $\mathrm{SnO}_{2}$. Next, $1 \mathrm{ml}$ of a perovskite solution [1.25 $\mathrm{M}$ in dimethylformamide (DMF) with a 1:1 molar ratio of lead iodide $\left(\mathrm{PbI}_{2}\right.$, Alfa Aesar, 99.9985\%) to methylammonium iodide (MAI with high purity, Xi'an Polymer Light Technology Corp, $\geq 99.5 \%$, MAI with low purity, Sigma-Aldrich, 98\%)] was mixed with $5 \mathrm{mg}$ of urea (Alfa Aesar, 98+\%) and then stirred at $60^{\circ} \mathrm{C}$ for $8 \mathrm{~h}$ in a glovebox. Afterward, the perovskite precursor solution was deposited on the $\mathrm{ITO} / \mathrm{SnO}_{2} / \mathrm{CPTA}$ template in a glovebox by spin-coating at $5000 \mathrm{rpm}$ for $30 \mathrm{~s}$, with chlorobenzene (CB) treatment $8 \mathrm{~s}$ after the spincoating began. Then, the film was annealed at $100^{\circ} \mathrm{C}$ for $10 \mathrm{~min}$. A hole transport solution was prepared by combining $1 \mathrm{ml}$ of a $72.3 \mathrm{mg} / \mathrm{ml}$ solution of $2,2^{\prime}, 7,7^{\prime}$-tetrakis( $N, N$-di- $p$-methoxyphenylamine) $9,9^{\prime}$-spirobifluorene (Spiro-OMeTAD) (Borun Chemicals, 99.7\%) in CB, $28.8 \mu \mathrm{l}$ of 4-tert-butylpyridine (Sigma-Aldrich, 96\%), and $17.5 \mu \mathrm{l}$ of a $520 \mathrm{mg} / \mathrm{ml}$ lithium bis(trifluoromethylsulfonyl)imide (Sigma-Aldrich, 99.9\%) stock solution in acetonitrile. A hole transport layer was spin-coated from the SpiroOMeTAD solution at $2000 \mathrm{rpm}$ for $30 \mathrm{~s}$, followed by deposition of a $75 \mathrm{~nm}$ patterned Au top electrode by thermal evaporation. All of the abovementioned solutions were filtered through a $0.45 \mu \mathrm{m}$ polytetrafluoroethylene filter prior to use.

\section{Characterization and measurements}

High-resolution transmission electron microscopy (HRTEM) images and selected area electron diffraction (SAED) patterns were obtained using a Philips Tecnai G2 
(a)
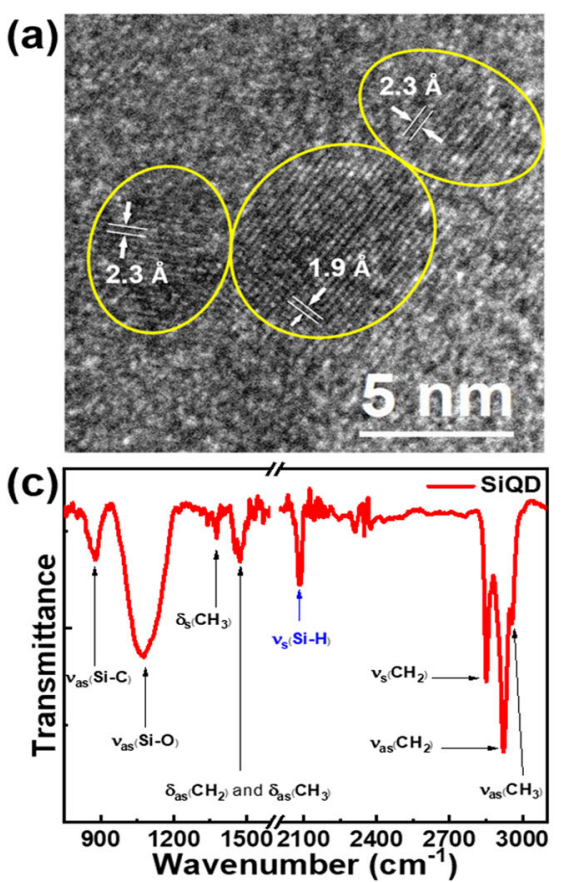

(b)
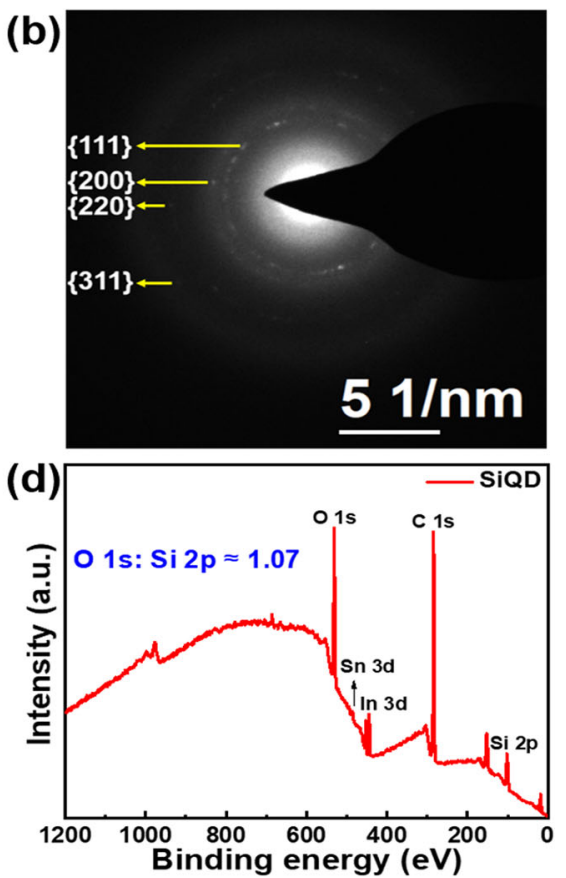

Fig. 1 Characterization of SiQDs. a HR-TEM image, b SAED pattern, c FTIR spectrum, and $\mathbf{d}$ survey XPS spectrum of as-synthesized SiQDS.

(FEI-TEM) microscope operating at $200 \mathrm{kV}$. Fourier transform infrared (FTIR) chemical analyses were performed with a NICOLET 6700 (Thermo Fisher Scientific, USA) instrument. X-ray photoelectron spectroscopy (XPS) measurements were recorded using a Kratos AXIS ULTRA DALD XPS/UPS system. XPS survey scans were collected to identify the overall surface composition using a monochromatic Al K $\alpha$ X-ray source $(1486.6 \mathrm{eV})$. The measurement procedures for the photocurrent density-voltage $(J-V)$ curves and the external quantum efficiency (EQE) spectra were as follows. The cell parameters were obtained under incident light with an intensity of $100 \mathrm{~mW} / \mathrm{cm}^{2}$ (as measured using a thermopile probe; Oriel 71964) generated by a $300 \mathrm{~W}$ solar simulator (Oriel Sol3A Class AAA Solar Simulator 9043A, Newport) and passed through an AM 1.5 filter (Oriel 74110). The light intensity was further calibrated using an Oriel reference solar cell (Oriel 91150) and adjusted to 1 sun. The monochromatic quantum efficiency was recorded using a monochromator (Oriel 74100) under short-circuit conditions. PL spectra were obtained using a fluorescence spectrophotometer (F-4600, Hitachi Ltd., Tokyo, Japan) with a $150 \mathrm{~W}$ Xe lamp as the excitation source at room temperature. UV/Visible transmission spectra were obtained using a Lambda 750 UV/VIS/NIR spectrophotometer (PerkinElmer). AFM (Hitachi AFM500II, tapping mode) was used to observe the surfaces of the perovskite and perovskite/SiQD films. Size distribution analysis via dynamic light scattering was performed with a
Nanotrac Wave (MicrotracBEL Corp., Japan) instrument. Ultraviolet photoelectron spectroscopy (UPS) measurements were conducted using a Shimadzu spectrometer (AXIS ULTRA DLD) with a HeI $(21.2 \mathrm{eV})$ discharge lamp.

\section{Results}

\section{Characterization of SiQDs}

SiQDs were synthesized mainly using a pulsed Nd:YAG laser for $266 \mathrm{~nm}$ irradiation of a porous $\mathrm{Si}$ suspension [consisting of porous Si powder, long unsaturated alkyl ligands (i.e., 1-decence) and HF] with minor modifications according to our previous results ${ }^{28,29}$ (see Supplementary Fig. 1 and the "Materials and methods" section). After laser fragmentation, the supernatant fluid was centrifuged at $13,000 \mathrm{rpm}$ for $20 \mathrm{~min}$ and then filtered through a membrane mesh (pore size circa $200 \mathrm{~nm}$ ). Finally, the filtered SiQDs were redispersed into toluene at a concentration of $10 \mathrm{mg} / \mathrm{ml}$ to form the target colloidal solution. Figure 1a shows an HR-TEM image of the asprepared colloidal SiQDs with diameters between approximately 4 and $7.5 \mathrm{~nm}$. These SiQDs were formed by fragmentation of porous $\mathrm{Si}$ powder, and thus, the size distribution of the SiQDs is similar to the size distribution of the $\mathrm{Si}$ cores constituting porous $\mathrm{Si}^{29}$. Since the porous $\mathrm{Si}$ cores have a relatively wide size distribution, the size distribution of the SiQDs is also wide (that is, $>5 \%$ of the average diameter). A dynamic light scattering measurement was performed, showing a colloidal hydrodynamic size distribution of $10.9 \pm 1.4 \mathrm{~nm}$ (see Supplementary Fig. 
2). The wide size distribution will benefit PSCs, which will be discussed later. Furthermore, in Fig. 1a, the visible crystal fringes of three SiQDs with two types of interplanar lattice distances of 1.9 and $2.3 \AA$ match the $\mathrm{Si}$ $(220)^{29}$ and $\mathrm{SiO}_{\mathrm{x}}(111)^{30}$ crystal planes, respectively, indicating the existence of oxide regions in the SiQDs. As presented in Fig. 1b, the SAED patterns indicate reflection bands for the $\{111\},\{220\}$, and $\{311\}$ index facets of the SiQDs, corresponding to the face-centered cubic (FCC) silicon phase with a lattice parameter of $5.43 \AA^{30}$. In addition, the $\{200\}$ planes in the SAED pattern are attributed to the crystal plane of FCC silicon with a smaller lattice parameter of $3.90 \AA^{30}$ due to the presence of silicon oxide, implying that the SiQDs are partially oxidized in air.

To introduce SiQDs into PSC devices, toluene-diluted $\mathrm{SiQD}$ suspensions with various concentrations $(0.001-1 \mathrm{mg} /$ $\mathrm{ml}$ ) were spin-coated onto perovskite layers at $4000 \mathrm{rpm}$ for $30 \mathrm{~s}$. After deposition of the SiQDs, the chemical structure of the SiQD films could be analyzed using the FTIR technique, as depicted in Fig. 1c. The FTIR spectrum of the assynthesized film is characterized by absorption bands attributable to the surface functionalities of the SiQDs after hydrosilylation, namely, the $\mathrm{Si}-\mathrm{O}$ asymmetric stretching mode $\left[v_{\text {as }}(\mathrm{Si}-\mathrm{O})\right]$ in the region from 1010 to $1080 \mathrm{~cm}^{-1}$ (refs. ${ }^{28,31}$ ), $v_{\text {as }}(\mathrm{Si}-\mathrm{C})$ at $864 \mathrm{~cm}^{-1}$ (ref. ${ }^{32}$ ), and the $\mathrm{Si}-\mathrm{H}$ symmetric stretching mode $\left[v_{\mathrm{s}}(\mathrm{Si}-\mathrm{H})\right]$ at $2100 \mathrm{~cm}^{-1}$ (ref. ${ }^{31}$ ). The as-synthesized SiQDs exhibit $\mathrm{Si}-\mathrm{O}$ vibrations in Fig. 1c due to oxidation of the silicon surface exposed to air, thus corroborating the HR-TEM and SAED results. The presence of the $\mathrm{Si}-\mathrm{C}$ band indicates that 1-decence molecules are directly attached to the $\mathrm{SiQD}$ surfaces via the hydrosilylation reaction. Herein, the $\mathrm{C}-\mathrm{H}$ bending modes $[\delta(\mathrm{C}-\mathrm{H})]$ and $\mathrm{C}-\mathrm{H}$ stretching vibrations $[v(\mathrm{C}-\mathrm{H})]$ located in regions $1200-1500 \mathrm{~cm}^{-1}$ (refs. ${ }^{28,31}$ ) and $2800-3000 \mathrm{~cm}^{-1}$ (ref. ${ }^{31}$ ), respectively, are from this long alkyl ligand. Figure $1 \mathrm{~d}$ plots a survey XPS spectrum to identify the oxygen content in the SiQDs. The XPS scan yielded an O 1s/Si $2 \mathrm{p}$ ratio of 1.07 , indicating that the SiQDs have a large oxidized proportion, in agreement with the abovementioned FTIR results.

\section{Photovoltaic performance}

The SiQD-containing PSC with the architecture of indium tin oxide (ITO) glass anode $/ \mathrm{SnO}_{2}$ electron transporter/CPTA electron transporter/ $\mathrm{CH}_{3} \mathrm{NH}_{3} \mathrm{PbI}_{3}$ perovskite/SiQDs/Spiro-OMeTAD hole transporter/Au cathode is schematically shown in Fig. 2a, wherein the $\mathrm{SiQDs}$ are used as photocurrent enhancers. Supplementary Fig. 3a shows a cross-sectional scanning electron microscopy (SEM) image of the pristine PSC device (without SiQDs); the perovskite absorber remained conformally flat over a wide range, with a film thickness of $320 \mathrm{~nm}$. Figure $2 \mathrm{~b}$ shows $J-V$ curves of PSCs without and with various concentrations of SiQDs (from 0.001 to $1 \mathrm{mg} / \mathrm{ml}$ ) under simulated AM $1.5 \mathrm{G}$ illumination $\left(100 \mathrm{~mW} / \mathrm{cm}^{2}\right)$. The detailed $J-V$ values are summarized in Table 1. These PSCs exhibit an open-circuit voltage $\left(V_{\mathrm{OC}}\right.$ ) of $1.07 \mathrm{~V}$ and similar fill factors (FFs) of $~ 79 \%$ up to the addition of excess SiQDs $(1 \mathrm{mg} / \mathrm{ml})$ to the PSC device. The champion PSC incorporating the photocurrentenhancing SiQDs at a concentration of $0.01 \mathrm{mg} / \mathrm{ml}$ yields a PCE of $19.6 \%$ with a short-circuit current density $\left(\mathrm{JC}_{\mathrm{SC}}\right)$ of $23.10 \mathrm{~mA} / \mathrm{cm}^{2}$. These two parameters are comparable to those of the control PSC without any SiQD coating, which shows a PCE of $18.2 \%$ and a $J_{\mathrm{SC}}$ of $21.44 \mathrm{~mA} / \mathrm{cm}^{2}$, indicating that the SiQDs can increase the efficiency of the PSC device through the superior photocurrent gain effect.

A small $J-V$ hysteresis is observed in the PSCs constructed with SiQDs in different scan directions, as shown in Fig. 2c. Fortunately, a stable photocurrent output is observed in Fig. 2d. After $1500 \mathrm{~s}$ of illumination, the SiQD-based PSC device still presents a high photocurrent of $21.20 \mathrm{~mA} / \mathrm{cm}^{2}$ with a stabilized PCE of $19.3 \%$ at the maximum power output (at $0.91 \mathrm{~V})$. Statistical $J-V$ results were collected by examining 15 PSCs from five batches, as depicted in Fig. $2 \mathrm{e}$ and given in Table 1. The same trend is observed, in which the PCE and $J_{\text {SC }}$ values of these PSCs are maximized when the SiQD concentration reaches $0.01 \mathrm{mg} / \mathrm{ml}$. Moreover, the increase in the PCEs is mainly due to the high photocurrent gain contributed by the SiQDs. In general, high-efficiency PSCs can be achieved by using high crystallinity perovskite layers. However, to fabricate these well-tailored perovskites, multistep sublimation of precursors is required, which greatly increases the costs in the PSC industry. Since SiQDs can harvest photon energy more effectively in PSCs, we applied this method to low-efficiency but inexpensive PSCs. SiQDs were added to a PSC device with a low-purity perovskite layer and remarkably suppressed the defect-induced photocarrier transport barrier, resulting in a PCE of $12.9 \%$ with a $J_{\mathrm{SC}}$ of $22.96 \mathrm{~mA} / \mathrm{cm}^{2}$ and an FF of $52.4 \%$ (see Supplementary Fig. 4). Compared with the bare PSC, the SiQD-based PSC exhibits a surprising increase in the PCE by $5.6 \%$ (from 7.3 to $12.9 \%$ ), indicating that SiQDs can provide another low-cost and effective way to promote PSCs in the commercial market.

\section{Photocurrent analysis}

From the above results, the efficiency improvement of the PSCs mainly comes from the additional photocurrent boost, and thus, the photocurrent gain can be further analyzed using the EQE. The EQE spectra of PSCs with various concentrations of SiQDs $(0.001-1 \mathrm{mg} / \mathrm{ml})$ and without SiQDs are depicted in Fig. 3a. The bestperforming PSC with $0.01 \mathrm{mg} / \mathrm{ml} \mathrm{SiQDs} \mathrm{shows} \mathrm{a} \mathrm{broad}$ EQE spectrum and a significantly high value of $>75 \%$ from 360 to $760 \mathrm{~nm}$, giving an integrated photocurrent of 
(a)

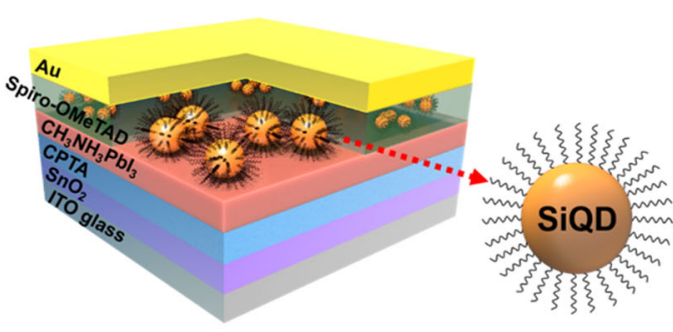

(c)

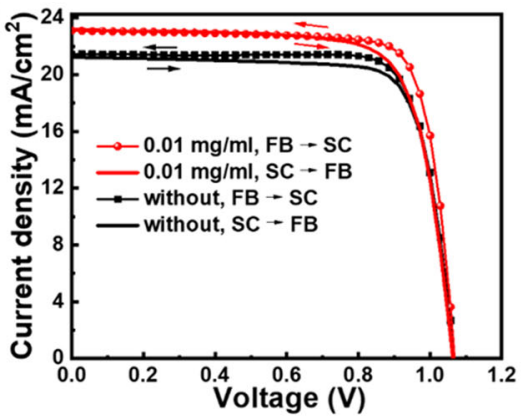

(d)

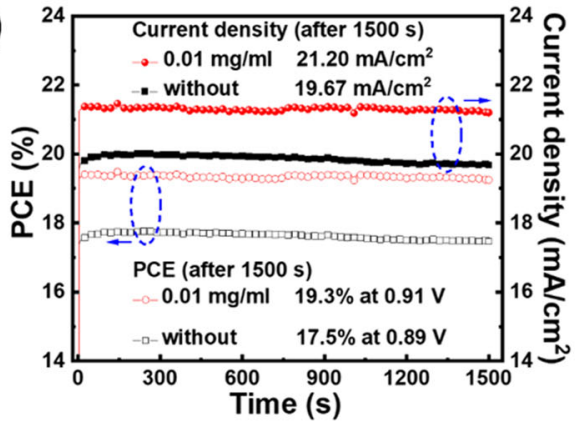

(b)

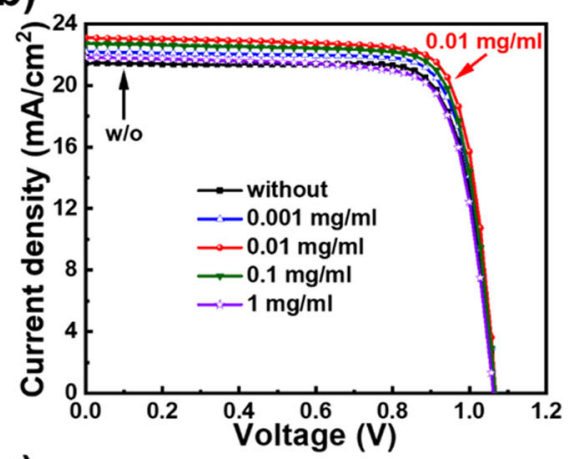

(e)

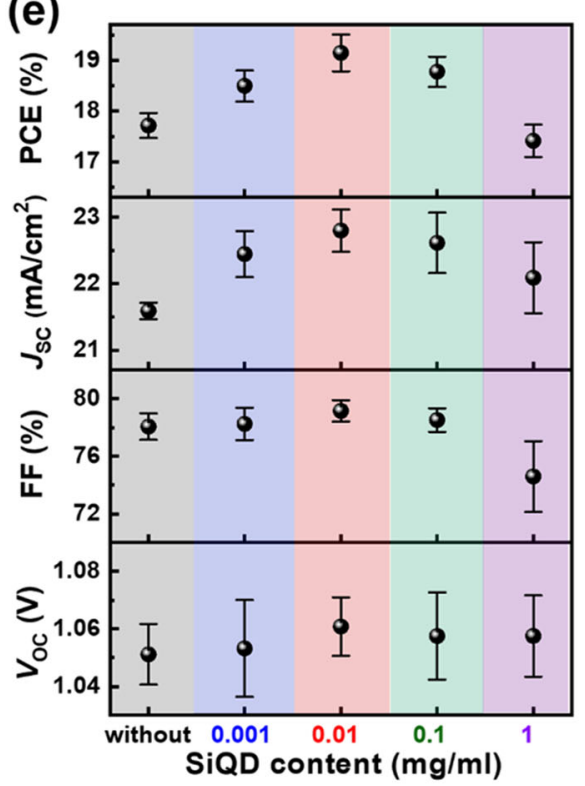

Fig. 2 Photovoltaic characterization. a Architecture of the SiQD-based PSC device. $\mathbf{b}$ J-V scans of PSCs with various amounts of SiQDs (0-1 mg/ml) under AM 1.5G illumination at $100 \mathrm{~mW} / \mathrm{cm}^{2}$. c J-V curves of the best-performing device $(0.01 \mathrm{mg} / \mathrm{ml})$ and control device (without SiQDs) measured in the FB to SC direction and back again under AM 1.5G illumination at $100 \mathrm{~mW} / \mathrm{cm}^{2}$. $\mathbf{d}$ Steady-state photocurrent density and power output at the maximum power point for the champion SiQD-based PSC (0.01 mg/ml) and control device (without SiQDs). e Standard deviations in the PCE, JSC, FF, and $V_{\mathrm{OC}}$ to evaluate the reproducibility, from the statistics of 15 devices each without or with different contents of SiQDs.

Table 1 Photovoltaic characteristics of PSCs incorporating SiQDs at various concentrations measured in the forward bias (FB) to short-circuit (SC) direction or in the SC to FB direction under AM $1.5 \mathrm{G}$ illumination $\left(100 \mathrm{~mW} / \mathrm{cm}^{2}\right)$.

\begin{tabular}{lllll}
\hline SiQD $(\mathbf{m g} / \mathbf{m l})$ & $V_{\text {OC }}(\mathbf{V})$ & $J_{\text {SC }}\left(\mathbf{m A} / \mathbf{c m}^{2}\right)$ & FF (\%) & PCE (\%) \\
\hline Without $(F B \rightarrow S C)$ & $1.07(1.05 \pm 0.01)$ & $21.44(21.59 \pm 0.13)$ & $79.4(78.1 \pm 0.9)$ & $18.2(17.7 \pm 0.2)$ \\
Without $(\mathrm{SC} \rightarrow \mathrm{FB})$ & 1.06 & 21.21 & 78.3 & 17.6 \\
$0.001(\mathrm{FB} \rightarrow \mathrm{SC})$ & $1.07(1.05 \pm 0.02)$ & $22.18(22.45 \pm 0.35)$ & $79.5(78.3 \pm 1.1)$ & $18.8(18.5 \pm 0.3)$ \\
$0.01(\mathrm{FB} \rightarrow \mathrm{SC})$ & $1.07(1.06 \pm 0.01)$ & $23.10(22.80 \pm 0.32)$ & $79.7(79.2 \pm 0.7)$ & $19.6(19.2 \pm 0.4)$ \\
$0.01(\mathrm{SC} \rightarrow \mathrm{FB})$ & 1.06 & 23.16 & 75.3 & 18.5 \\
$0.1(\mathrm{FB} \rightarrow \mathrm{SC})$ & $1.07(1.06 \pm 0.02)$ & $22.72(22.62 \pm 0.46)$ & $79.3(78.5 \pm 0.8)$ & $19.2(18.8 \pm 0.3)$ \\
$1(\mathrm{FB} \rightarrow \mathrm{SC})$ & $1.06(1.06 \pm 0.01)$ & $21.82(22.09 \pm 0.54)$ & $77.5(74.6 \pm 2.5)$ & $17.9(17.4 \pm 0.3)$ \\
\hline
\end{tabular}

The average results in brackets represent the standard deviation of 15 devices from five different batches. 

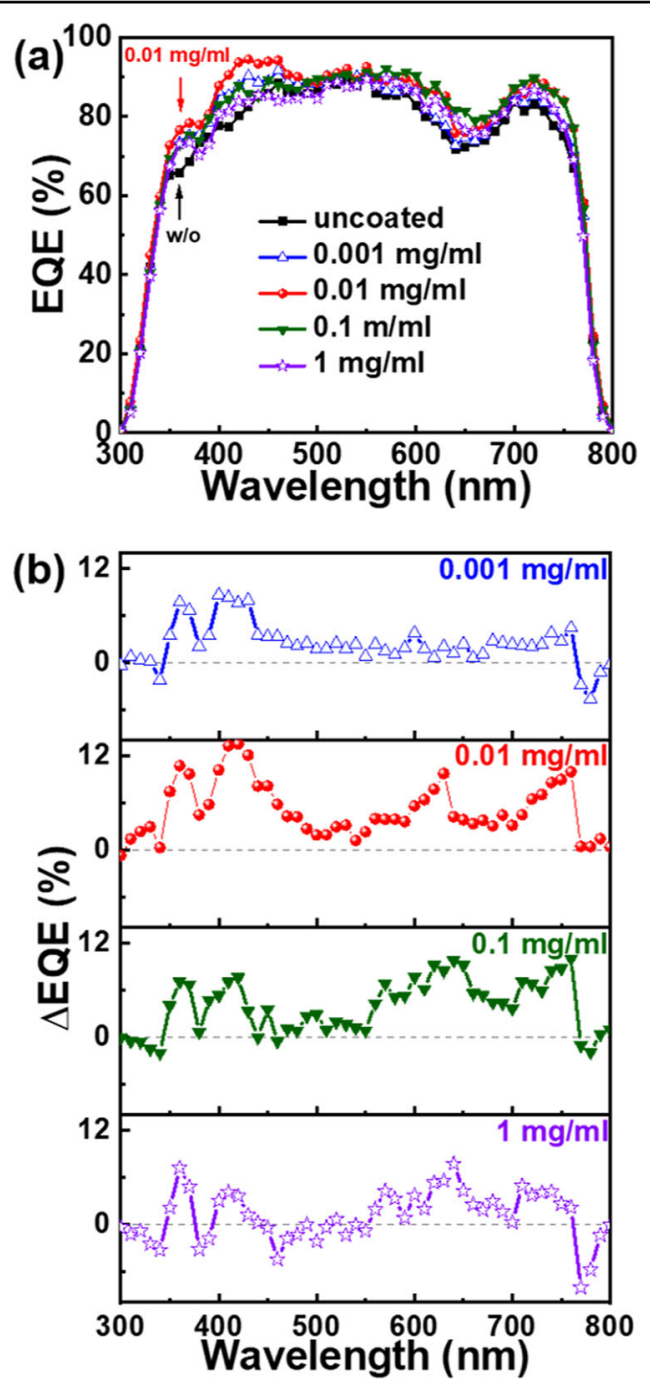

Fig. 3 EQE curves for PSCs. a EQE spectra of PSCs without and with different concentrations of SiQDs. $\mathbf{b}$ EQE enhancement spectra of PSCs with $0.001,0.01,0.1$, and $1 \mathrm{mg} / \mathrm{ml}$ SiQDs.

$21.98 \mathrm{~mA} / \mathrm{cm}^{2}$. This value is consistent with the $J_{\mathrm{SC}}$ parameter measured from the $J-V$ characteristics under simulated AM 1.5G irradiation (see Fig. 2b). Furthermore, the corresponding EQE enhancements were extracted using the EQEs of SiQD-based PSCs divided by those from the EQE spectrum of the reference PSC (without SiQDs) ${ }^{33}$, as shown in Fig. 3b. The enhancement in the EQE ( $\triangle \mathrm{EQE})$ demonstrates that the deposition of $0.01 \mathrm{mg} / \mathrm{ml} \mathrm{SiQDs}$ can remarkably improve the photocurrent generation efficiency, with EQE enhancement peaks of more than $10 \%$ at $360,410,630$, and $758 \mathrm{~nm}$. We also provide two other sets of EQE and $\triangle \mathrm{EQE}$ data, as shown in Supplementary Figs. 5 and 6, respectively. The results show that PSCs with $0.01 \mathrm{mg} / \mathrm{ml} \mathrm{SiQDs} \mathrm{still} \mathrm{have}$ the highest EQE gain effect. Once the PSC is covered by SiQDs, its EQE improvement effect at short wavelengths is obvious. At the same time, when the concentration of SiQDs spin-coated on the PSC device exceeds $0.01 \mathrm{mg} / \mathrm{ml}$, the EQE improvement in the visible band also increases. The results show that SiQD-based PSC devices exhibit photocurrent gain effects over a wide range from UV to visible light.

\section{Discussion}

\section{Photon management in SiQD-based PSCs}

To gain insight into the photon management mechanism, the PL signal from SiQDs was studied in detail using fluorescence mapping spectra, as demonstrated in Fig. 4a. Two emission bands of SiQDs are displayed in the visible wavelength range from 380 to $775 \mathrm{~nm}$ under excitation at UV-visible regions $(300-530 \mathrm{~nm})$. The long wavelength luminescence can be attributed to quantum confinement effects $^{34-36}$, while the short wavelength luminescence is caused by oxidized SiQDs ${ }^{27,37}$. Placing SiQDs in air to measure the PL signal may cause some of them to be oxidized. Fortunately, the oxidation of SiQDs only occurs on surfaces without ligands ${ }^{37}$. Once these ligand-free surfaces are oxidized, the oxidation of SiQD stops. The PL signal of $\mathrm{SiQD}$ shown in Fig. 4a is the data after the oxidation has stopped. The luminescence of these two bands (see Fig. 4a) can be reabsorbed by perovskite crystals and converted into electrical energy. Figure $4 \mathrm{~b}$ clearly shows that SiQDs absorb photons from 300 to $530 \mathrm{~nm}$ and emit visible photons $(380-775 \mathrm{~nm})$ after the SiQDs are coated onto the perovskite $\left[\mathrm{CH}_{3} \mathrm{NH}_{3} \mathrm{PbI}_{3}\right.$ $\left.\left(\mathrm{MAPbI}_{3}\right)\right]$ surfaces. Then, the visible emission can be reabsorbed by $\mathrm{MAPbI}_{3}$ light harvesters. PSC photovoltaics can only effectively convert sunlight in the $400-750 \mathrm{~nm}$ spectral range into electricity ${ }^{38-40}$. Fortunately, the SiQDs show an excellent UV-visible absorption capacity (see Supplementary Fig. 7) and can convert short wavelength light to a perovskite-operating visible band. In addition, the size distribution of the SiQDs is relatively wide (see Supplementary Fig. 2). Therefore, a broad excitation peak is achieved between 300 and $530 \mathrm{~nm}$ due to quantum size effects $^{35,36}$. As a result, the photoelectric conversion efficiency across this broad UV-visible region will be more efficient in PSCs. Therefore, the EQE responses of all SiQD-based PSCs are enhanced at short wavelengths, as shown in Fig. 3b.

Moreover, the EQEs of SiQD-containing PSCs in the $550-770 \mathrm{~nm}$ wavelength region are also higher than those of the PSC without SiQDs (see Fig. 3a). The optical properties were further analyzed using UV-visible reflection spectra. After coating the $\mathrm{MAPbI}_{3}$ layer with SiQDs, the reflection response was significantly improved at wavelengths between 550 and $800 \mathrm{~nm}$, as shown in Fig. 4c. The strong reflection caused by SiQDs is beneficial for reabsorbing the visible light that the perovskite does not absorb. In addition, the reflection intensity in the $550-800 \mathrm{~nm}$ band 
(a)

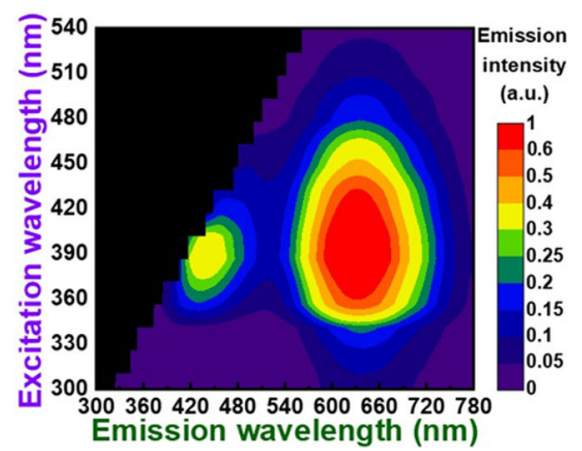

(b)

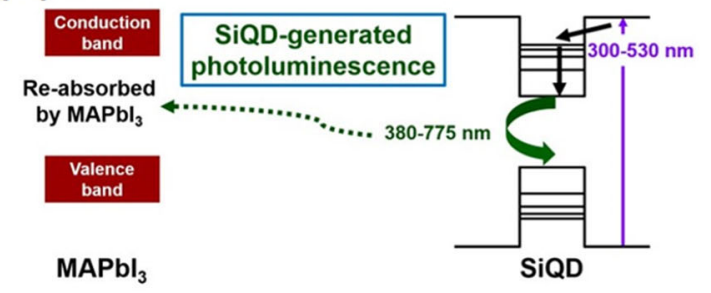

(c)

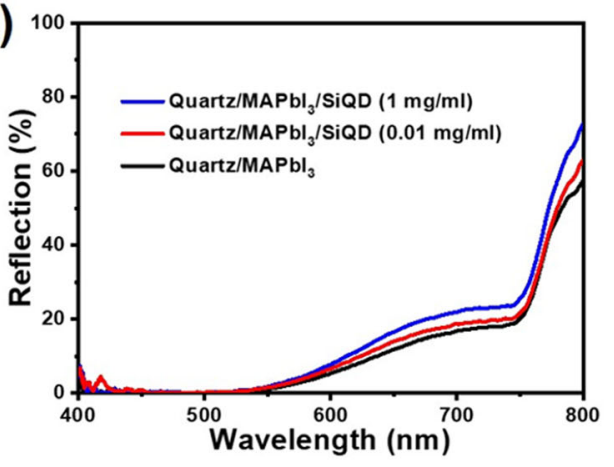

Fig. 4 Photocarrier dynamics at $\mathrm{MAPbl}_{3} / \mathrm{SiQD}$ heterojunctions. a Fluorescence mapping spectra of SiQDs. $\mathbf{b}$ Schematic illustration of quantum-assisted sunlight reabsorption processes at $\mathrm{MAPb}_{3} / \mathrm{SiQD}$ interfaces. c Reflection spectra of quartz/MAPbl 3 and quartz/MAPb| ${ }_{3} /$ SiQD samples.

is enhanced as the concentration of SiQDs coated on the perovskite increases. This result is consistent with the $\triangle \mathrm{EQE}$ signals (see Fig. 3b). When the SiQD concentration of the SiQD-based PSC samples is increased from 0.001 to $0.1 \mathrm{mg} / \mathrm{ml}$, the corresponding $\triangle \mathrm{EQE}$ in the $550-800 \mathrm{~nm}$ range is improved. However, the EQE response requires not only the capability of the material to absorb light but also the ability to convert light into electricity. Although the sample with a SiQD concentration of $1 \mathrm{mg} / \mathrm{ml}$ has a stronger reflection spectrum than the $0.01 \mathrm{mg} / \mathrm{ml}$ sample, its $\triangle E Q E$ response at $550-800 \mathrm{~nm}$ is decreased. This shows that the photoelectric conversion ability has a great impact after deposition of a high concentration of SiQDs. Thus, enhancement of the EQE in the long wavelength visible region $(>550 \mathrm{~nm})$ can be obtained by covering the perovskite crystals with SiQDs (see Fig. 4b). Because of these two SiQD-assisted photon management effects, namely, converting short wavelength light into visible light and reflecting long wavelength visible light, the EQE response of the best-performing PSC device $(0.01 \mathrm{mg} / \mathrm{ml})$ can be enhanced across a $400 \mathrm{~nm}$ ultrawide region $(\sim 350-750 \mathrm{~nm})$ (see Fig. 4b). In addition, the traditional passivation layer deposited after the perovskite is used to promote the carrier transport of defective perovskite crystals (including defects, grain boundaries and incomplete crystals). In this work, SiQDs are mainly used to capture the UV-visible light unabsorbed by the perovskite and then recycle this light through reabsorption and reflection. Since SiQD-based PSCs capture more light than the pristine device (without SiQDs), a higher photocurrent will be generated. In these PSCs, the upper SiQD clusters do not change or passivate the bottom perovskite crystals. Therefore, the SiQDs are a photocurrent enhancer, not a passivator (as confirmed in Supplementary Fig. 8).

\section{Morphology of SiQDs and the photoelectric impacts on PSCs}

To further explore the reasons for the reduction in the photoelectric responses (including the $J-V$ curves and EQE characteristics) after adding excess SiQDs (concentration $>1 \mathrm{mg} / \mathrm{ml}$ ), we systematically studied the morphology evolution of SiQDs deposited onto $\mathrm{MAPbI}_{3}$ perovskites with increasing concentration. Figure 5a presents atomic force microscopy (AFM) images of the pristine $\mathrm{MAPbI}_{3}$ perovskite (without SiQDs) and of SiQDs spin-coated on perovskite crystals at concentrations of 0.01 and $1 \mathrm{mg} / \mathrm{ml}$. Compared with the pristine perovskite crystals that exhibit clear lattice fringes, the $\mathrm{MAPbI}_{3}$ coated with $0.01 \mathrm{mg} / \mathrm{ml} \mathrm{SiQDs}$ appears to have different size particles on the perovskite crystals. As shown in Supplementary Fig. 3b, c, the AFM depth profiles show that the thicknesses of the $0.01 \mathrm{mg} / \mathrm{ml}$ SiQDs and $1 \mathrm{mg} / \mathrm{ml} \mathrm{SiQDs}$ are 12.7 and $15.2 \mathrm{~nm}$, respectively. The SiQD clusters are approximately tens of nanometers in diameter, which are much larger than the as-prepared SiQDs (see Fig. 1a). This phenomenon occurs because the surface of the as-synthesized SiQDs has a long carbon chain, and once exposed to the hydrophilic perovskite surface, these SiQDs will aggregate into clusters to reduce the interface energy. The survey XPS spectrum demonstrates a $\mathrm{Si} 2 \mathrm{p} / \mathrm{Pb} 4 \mathrm{f}$ value of $\sim 13.5 \%$ (see Supplementary Fig. 9); hence, the amount of SiQDs in the perovskite/SiQD $(0.01 \mathrm{mg} / \mathrm{ml})$ sample can be quantified. After a large amount of SiQDs was deposited onto the perovskite $(1 \mathrm{mg} / \mathrm{ml})$, the perovskite surface was covered by SiQD clusters, resulting in the disappearance of the perovskite grains. These samples (without SiQDs, 0.01 and $1 \mathrm{mg} / \mathrm{ml}$ ) are plotted as 

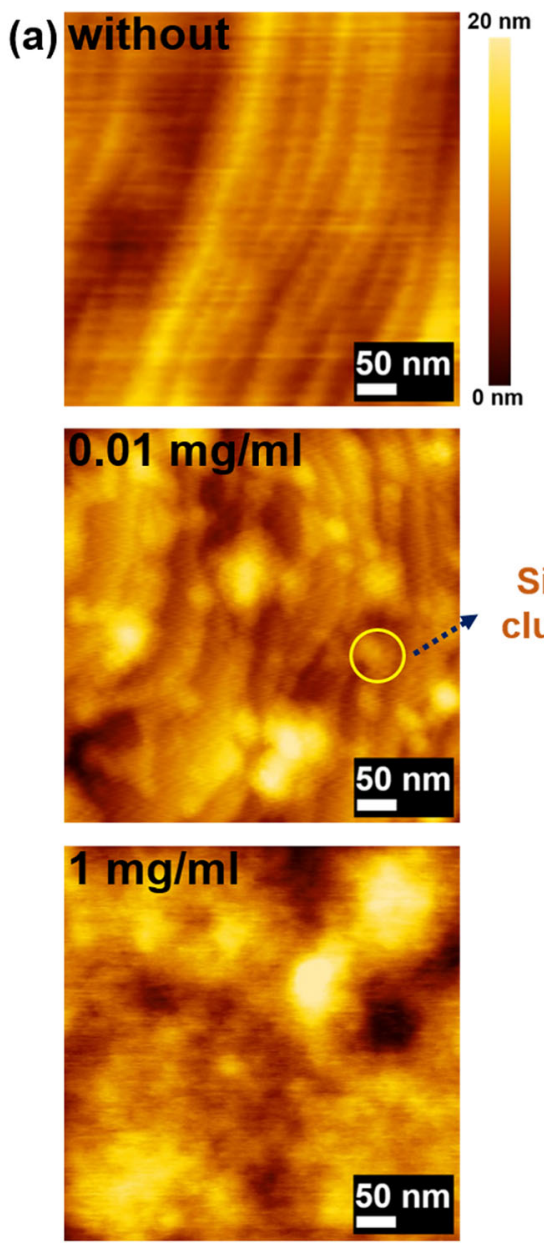

(b)

without

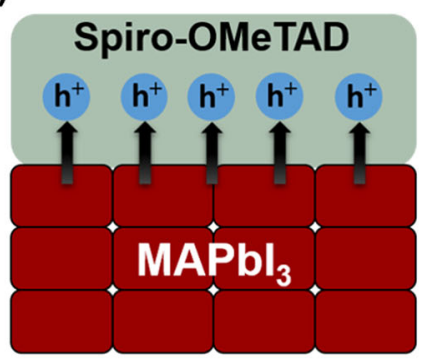

$0.01 \mathrm{mg} / \mathrm{ml}$

Spiro-OMeTAD

SIQD cluster

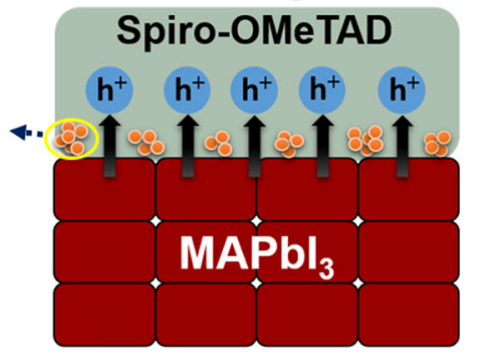

$1 \mathrm{mg} / \mathrm{ml}$

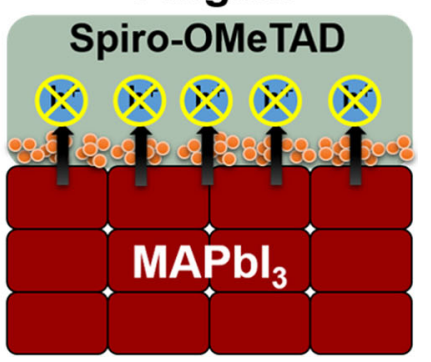

Fig. 5 Distribution of SiQDs on $\mathrm{MAPbl}_{3}$ perovskite grains and their impact on hole transfer. a AFM images of ITO glass/SnO $/ 2$ CPTA/MAPbl 3 (without SiQDs), ITO glass $/ \mathrm{SnO}_{2} / \mathrm{CPTA} / \mathrm{MAPbl}_{3} / \mathrm{SiQDs}(0.01 \mathrm{mg} / \mathrm{ml})$, and ITO glass $/ \mathrm{SnO}_{2} / \mathrm{CPTA} / \mathrm{MAPbl}_{3} / \mathrm{SiQDs}(1 \mathrm{mg} / \mathrm{ml})$. b Schematic depiction of the effect of different SiQD concentrations on the hole $\left(\mathrm{h}^{+}\right)$transfer at $\mathrm{MAPb}_{3} / \mathrm{SiQD}$ cluster/Spiro-OMeTAD heterojunctions.

corresponding side schematics to explain the influence on the photocarrier dynamics of the SiQD morphologies, as shown in Fig. 5b. In the pristine perovskite (without SiQDs) and the low SiQD concentration sample $(0.01 \mathrm{mg} / \mathrm{ml})$, perovskite-generated holes can be directly transported to the hole transport layer [Spiro-OMeTAD] because most perovskite grains are exposed. Although the valence band level of the SiQDs (see Supplementary Fig. 10) is lower than that of $\mathrm{MAPbI}_{3}$, hole transport in the SiQD-based PSC is still easy. However, as the concentration of SiQDs increases to $1 \mathrm{mg} / \mathrm{ml}$, the holes will be blocked due to the lower valence band level of SiQDs with high coverage. This will cause hole accumulation at the perovskite/SiQD interface, leading to poor performance of PSCs with many SiQDs. These results illustrate that appropriate adjustment of the SiQD amount can improve the PCEs, FFs, and $J_{\mathrm{SC}}$ of PSCs without excessively increasing the cost.

\section{Conclusion}

In summary, we have successfully introduced SiQDs into PSC devices to achieve a PCE enhancement up to $1.4 \%$ (increased from 18.2 to $19.6 \%$ ). SiQDs with a relatively wide size distribution were synthesized using pulsed UV laser irradiation, yielding a broad excitation peak from 300 to $530 \mathrm{~nm}$. After excitation at broad UVvisible regions, the SiQDs converted the short wavelength photons into perovskite-absorbable visible bands. At the same time, reflection occurred at the perovskite/ SiQD interface, resulting in $550-800 \mathrm{~nm}$ perovskiteunabsorbed light being reflected and then reabsorbed by the perovskite crystals. Through these sunlight harvesting methods, UV light and unabsorbed visible light can be effectively utilized by the PSCs, achieving a high $J_{\mathrm{SC}}$ of $23.10 \mathrm{~mA} / \mathrm{cm}^{2}$, and these phenomena are economically beneficial to low-purity perovskite-based PSCs, with an impressive PCE boost of up to $5.6 \%$ (increased from 
7.3 to $12.9 \%)$. In general, low-purity perovskites lose too much light due to the poor crystallinity. Once SiQDs are coated onto the surface of low-quality perovskites, a large amount of unabsorbed light will be reused. For high-purity perovskites, since most of the light is absorbed by these perovskites, the ratio of recovered light is relatively low. Therefore, the SiQDs are more effective for low-purity PSCs than for high-purity PSCs. Generally, perovskite crystals require high-purity precursors and strict process control to achieve high crystallinity characteristics. If SiQDs are introduced into inexpensive low-purity perovskite solar cells, this will provide a new way to commercialize PSCs. Therefore, the photocurrent management strategy has tremendous implications for the real application of low-cost, highefficiency PSCs.

\section{Acknowledgements}

Y.-C.W. acknowledges the financial support from JSPS-KAKENHI (Project No. 20K15135) and the ICYS, NIMS for research funding. C.-W.C. acknowledges the financial support from the Minster of Science and Technology (MOST), Taiwan (Project No. 108-2923-M-002-002-MY2, MOST-NIMS add-on project). Y.-C.W. and K.T. thank Dr. Takatsugu Wakahara (NIMS), Prof. Keiji Ueno (Saitama University) and Prof. Ryo Ishikawa (Saitama University) for useful discussions and support in the experiment. Y.-C.W. and K.T. also thank Dr. Tsuneji Sakai (MicrotracBEL Corp.) for helpful particle analysis. Financial support from the Center of Atomic Initiative for New Materials (Al-Mat), National Taiwan University, from the Featured Areas Research Center Program within the framework of the Higher Education Sprout Project by the Ministry of Education in Taiwan (108L9008), is also acknowledged.

\section{Author details}

${ }^{1}$ International Center for Young Scientists (ICYS) \& WPI International Center for Materials Nanoarchitectonics (WPI-MANA), National Institute for Materials Science (NIMS), Tsukuba, Ibaraki 305-0044, Japan. ${ }^{2}$ Department of Materials Science and Engineering, National Taiwan University, Taipei 10617, Taiwan. ${ }^{3}$ Department of Electrical and Electronic Engineering, Hosei University, Koganei, Tokyo 184-8584, Japan. ${ }^{4}$ Department of Chemistry, Tunghai University, Taichung 40704, Taiwan. ${ }^{5}$ Quantum14, Ltd., Higashi-Koganei Jigyosozo Center, Koganei, Tokyo 184-0002, Japan. ${ }^{6}$ Center of Atomic Initiative for New Materials (AI-MAT), National Taiwan University, Taipei 10617, Taiwan

\section{Author contributions}

Y.-C.W., C.-W.C., and K.T. conceived and supervised this project. Y.-C.W. carried out the final device optimization. T.N., N.K., and T.S. synthesized the colloidal SiQDs. Y.-C.W., S.-K.H., T.N., Y.-T.K., C.-H.C., D.-Y.W., Y.J.C., and S.L. performed the data collection, interpretation, and analysis. Y.-C.W., C.-W.C., and K.T. wrote and revised the paper. All authors commented on and agreed to its content.

\section{Conflict of interest}

The authors declare that they have no conflict of interest.

\section{Publisher's note}

Springer Nature remains neutral with regard to jurisdictional claims in published maps and institutional affiliations.

Supplementary information is available for this paper at https://doi.org/ 10.1038/s41427-020-00236-1.

Received: 17 April 2020 Revised: 5 June 2020 Accepted: 8 June 2020. Published online: 28 August 2020

\section{References}

1. Jena, A. K., Kulkarni, A. \& Miyasaka, T. Halide perovskite photovoltaics: background, status, and future prospects. Chem. Rev. 119, 3036-3103 (2019).

2. Jeon, N. J. et al. A fluorene-terminated hole-transporting material for highly efficient and stable perovskite solar cells. Nat. Energy 3, 682-689 (2018).

3. Rong, Y. et al. Challenges for commercializing perovskite solar cells. Science 361, eaat8235 (2018).

4. Lu, Y.-A. et al. Coral-like perovskite nanostructures for enhanced lightharvesting and accelerated charge extraction in perovskite solar cells. Nano Energy 58, 138-146 (2019).

5. Bai, S. et al. Planar perovskite solar cells with long-term stability using ionic liquid additives. Nature 571, 245-250 (2019).

6. Pisoni, S. et al. Tailored lead iodide growth for efficient flexible perovskite solar cells and thin-film tandem devices. NPG Asia Mater. 10, 1076-1085 (2018).

7. Li, S.-S. et al. Intermixing-seeded growth for high-performance planar heterojunction perovskite solar cells assisted by precursor-capped nanoparticles. Energy Environ. Sci. 9, 1282-1289 (2016).

8. Wang, Y.-C. et al. Electron-transport-layer-assisted crystallization of perovskite films for high-efficiency planar heterojunction solar cells. Adv. Funct. Mater. 28, 1706317 (2018).

9. Shirayama, M. et al. Optical transitions in hybrid perovskite solar cells: ellipsometry, density functional theory, and quantum efficiency analyses for $\mathrm{CH}_{3} \mathrm{NH}_{3} \mathrm{Pbl}_{3}$. Phys. Rev. Appl. 5, 014012 (2016).

10. Best Research-Cell Efficiencies. https://www.nrel.gov/pv/cell-efficiency.html (NREL, 2019).

11. Extance, A. The reality behind solar power's next star material. Nature $\mathbf{5 7 0}$, 429-432 (2019).

12. Leijtens, T., Bush, K. A., Prasanna, R. \& McGehee, M. D. Opportunities and challenges for tandem solar cells using metal halide perovskite semiconductors. Nat. Energy 3, 828-838 (2018).

13. Bush, K. A. et al. 23.6\%-efficient monolithic perovskite/silicon tandem solar cells with improved stability. Nat. Energy 2, 17009 (2017).

14. Sahli, F. et al. Fully textured monolithic perovskite/silicon tandem solar cells with 25.2\% power conversion efficiency. Nat. Mater. 17, 820-826 (2018).

15. Chen, B. et al. Grain engineering for perovskite/silicon monolithic tandem solar cells with efficiency of 25.4\%. Joule 3, 177-190 (2019).

16. Green, M. A. \& Bremner, S. P. Energy conversion approaches and materials for high-efficiency photovoltaics. Nat. Mater. 16, 23-34 (2017).

17. Nayak, P. K., Mahesh, S., Snaith, H. J. \& Cahen, D. Photovoltaic solar cell technologies: analysing the state of the art. Nat. Rev. Mater. 4, 269-285 (2019).

18. Duong, T. et al. Rubidium multication perovskite with optimized bandgap for perovskite-silicon tandem with over 26\% efficiency. Adv. Energy Mater. 7, 1700228 (2017).

19. Zhang, W. et al. Enhancement of perovskite-based solar cells employing coreshell metal nanoparticles. Nano Lett. 13, 4505-4510 (2013).

20. Svrcek, $V$. et al. A silicon nanocrystal/polymer nanocomposite as a downconversion layer in organic and hybrid solar cells. Nanoscale 7, 11566-11574 (2015).

21. Maier-Flaig, F. et al. Multicolor silicon light-emitting diodes (SiLEDs). Nano Lett. 13, 475-480 (2013).

22. Priolo, F., Gregorkiewicz, T., Galli, M. \& Krauss, T. F. Silicon nanostructures for photonics and photovoltaics. Nat. Nanotechnol. 9, 19-32 (2014).

23. Meinardi, F. et al. Highly efficient luminescent solar concentrators based on earth-abundant indirect-bandgap silicon quantum dots. Nat. Photonics 11, 177-185 (2017).

24. Canham, L. T. Silicon quantum wire array fabrication by electrochemical and chemical dissolution of wafers. Appl. Phys. Lett. 57, 1046-1048 (1990).

25. Fauchet, P. M. Light emission from Si quantum dots. Mater. Today 8, 26-33 (2005).

26. Mizuno, H., Koyama, H. \& Koshida, N. Oxide-free blue photoluminescence from photochemically etched porous silicon. Appl. Phys. Lett. 69, 3779-3781 (1996).

27. Dohnalová, K. et al. Surface brightens up Si quantum dots: direct bandgap-like size-tunable emission. Light Sci. Appl. 2, e47 (2013).

28. Yuan, Z., Nakamura, T., Adachi, S. \& Matsuishi, K. Improvement of laser processing for colloidal silicon nanocrystal formation in a reactive solvent. J. Phys. Chem. C. 121, 8623-8629 (2017).

29. Nakamura, T., Yuan, Z., Watanabe, K. \& Adachi, S. Bright and multicolor luminescent colloidal Si nanocrystals prepared by pulsed laser irradiation in liquid. Appl. Phys. Lett. 108, 023105 (2016). 
30. Saxena, N., Kumar, P., Agarwal, A. \& Kanjilal, D. Lattice distortion in ion beam synthesized silicon nanocrystals in SiOx thin films. Phys. Status Solidi A 209 283-288 (2012).

31. Hua, F., Erogbogbo, F., Swihart, M. T. \& Ruckenstein, E. Organically capped silicon nanoparticles with blue photoluminescence prepared by hydrosilylation followed by oxidation. Langmuir 22, 4363-4370 (2006).

32. Esteves, A. C. C. et al. Influence of cross-linker concentration on the crosslinking of PDMS and the network structures formed. Polymer 50, 3955-3966 (2009).

33. Tsai, M.-L. et al. Efficiency enhancement of silicon heterojunction solar cells via photon management using graphene quantum dot as downconverters. Nano Lett. 16, 309-313 (2016).

34. Chan, M. Y. \& Lee, P. S. Fabrication of silicon nanocrystals and its room temperature luminescence effects. Int. J. Nanosci. 5, 565-570 (2006).

35. Sun, W. et al. Switching-on quantum size effects in silicon nanocrystals. Adv. Mater. 27, 746-749 (2015).
36. Mastronardi, M. L. et al. Size-dependent absolute quantum yields for sizeseparated colloidally-stable silicon nanocrystals. Nano Lett. 12, 337-342 (2012).

37. Gupta, A., Swihart, M. T. \& Wiggers, H. Luminescent colloidal dispersion of silicon quantum dots from microwave plasma synthesis: exploring the photoluminescence behavior across the visible spectrum. Adv. Funct. Mater. 19, 696-703 (2009).

38. Wang, Q. et al. Energy-down-shift $\mathrm{CsPbCl}_{3}: \mathrm{Mn}$ quantum dots for boosting the efficiency and stability of perovskite solar cells. ACS Energy Lett. 2, 1479-1486 (2017).

39. Wang, Y.-C. et al. Efficient and hysteresis-free perovskite solar cells based on a solution processable polar fullerene electron transport layer. Adv. Energy Mater. 7, 1701144 (2017).

40. Zhao, D. et al. High-efficiency solution-processed planar perovskite solar cells with a polymer hole transport layer. Adv. Energy Mater. 5, 1401855 (2015). 